\title{
Fractional Generalizations of Zakai Equation and Some Solution Methods
}

\author{
Sabir Umarov \\ University of New Haven, sumarov@newhaven.edu \\ Fred Daum \\ Raytheon \\ Kenric Nelson \\ Boston University
}

Follow this and additional works at: https://digitalcommons.newhaven.edu/mathematics-facpubs

Part of the Mathematics Commons

\section{Publisher Citation}

Umarov, S., Daum, F. \& Nelson, K. (2018). Fractional generalizations of Zakai equation and some solution methods. Fractional Calculus and Applied Analysis, 21(2), pp. 336-353.

\section{Comments}

MSC 2010: Primary 60G35; Secondary 35R11, 93E10, 60G05, 35Q84

This is the final version of the article published in

Fract. Calc. Appl. Anal., 21(2), pp. 336-353, watermarked for non-commercial sharing by the author. The original publication is located at http://dx.doi.org/10.1515/fca-2018-0020 


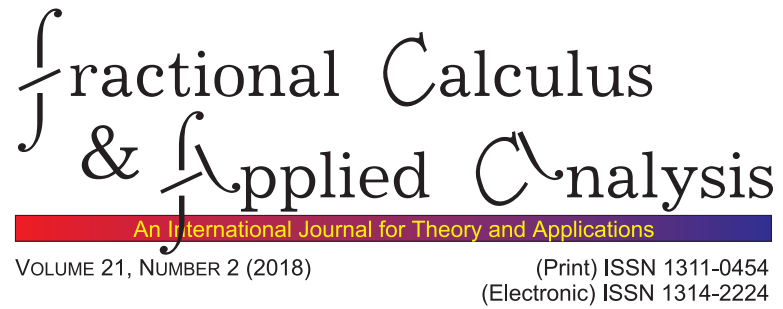

\title{
RESEARCH PAPER
}

\author{
FRACTIONAL GENERALIZATIONS OF \\ ZAKAI EQUATION AND SOME \\ SOLUTION METHODS
}

\author{
Sabir Umarov ${ }^{1}$, Fred Daum ${ }^{2}$, Kenric Nelson ${ }^{3}$
}

\begin{abstract}
The paper discusses fractional generalizations of Zakai equations arising in filtering problems. The derivation of the fractional Zakai equation, existence and uniqueness of its solution, as well as some methods of solution to the fractional filtering problem, including fractional version of the particle flow method, are presented.

MSC 2010: Primary 60G35; Secondary 35R11, 93E10, 60G05, 35Q84

Key Words and Phrases: fractional filtering, fractional Zakai equation, fractional Fokker-Planck-Kolmogorov equation, particle flow
\end{abstract}

\section{Introduction}

One of Albert Einstein's Annus Mirabilis 1905 papers [6] was devoted to the theoretical explanation of the Brownian motion. A little earlier (in 1900) Bachelier published his doctoral dissertation "Théorie de la spéculation" ("The Theory of Speculation") modeling Brownian motion from the economics point of view. In 1908 Langevin published his work with a stochastic differential equation which was "understood mathematically" only after a stochastic calculus was introduced by Itô in 1944-48. The Fokker-Planck equation, a deterministic form of describing the dynamics of a random process in terms of transition probabilities, was invented in 191317. Its complete "mathematical understanding" become available after

(c) 2018 Diogenes Co., Sofia pp. 336-353, DOI: 10.1515/fca-2018-0020

DE GRUYTER 
the appearance of the distribution (generalized function) theory (Sobolev (1938), Schwartz (1951)) and was embodied in Kolmogorov's backward and forward equations. Today the triple relationship between Brownian motion, Itô stochastic differential equations driven by Brownian motion and their associated Fokker-Planck-Kolmogorov (FPK) partial differential equations is well known.

In 1961 Kalman and Bucy solved a linear filtering problem, which is (mathematically) a wide generalization of the above concept. Namely, in the filtering problem one is interested in a stochastic process under additional information obtained from observation/measurement. As a result, FPK counterpart of the filtering process is not deterministic, but is a stochastic partial differential equation. The latter is called a Zakai equation, which was first derived by Zakai [26] in 1969.

This paper is devoted to fractional generalizations of the relationship between the driving process, corresponding SDEs, and associated fractional FPK and Zakai equations. For simplicity, we restrict our considerations to the case of time-changed Brownian motion, as the driving process. The driving process of a stochastic differential equation plays a key role in the evolution of the solution to the corresponding SDE. The processes associated with fractional order FPK and Zakai equations are usually driven by complex processes. Even in the simplest case of the fractional KFP equation $\partial^{\beta} u=\Delta u$, where $\Delta$ is the Laplace operator, and $\partial^{\beta}$ is a fractional derivative of order $0<\beta<1$, which is the case of time-changed Brownian motion, the driving process is a semimartingale, but not a Lévy process.

In the last few decades, fractional FPK type equations have been used in modeling various complex processes in physics, finance, hydrology, cell biology, etc. Complexity includes phenomena such as weak or strong correlations, different sub- or super-diffusive modes, memory and jump effects. For example, experimental studies of the motion of proteins or other macromolecules in a cell membrane show apparent subdiffusive motion with several simultaneous diffusive modes. In various engineering applications one has additional information available continuously due to measurements/observations, and the best estimate of the state process using this additional information is of interest.

Fractional Zakai type equations describe filtering problems whose state and observation processes are driven by a time-changed Brownian motion (or other standard driving processes). In Sections 34 4 we will show a derivation of the fractional Zakai equation, in which the time change process is the inverse to a Lévy stable subordinator with the stability index $\beta \in(0,1)$ and discuss existence and uniqueness of a solution, as well as some methods of solution. 


\section{Auxiliaries}

Fractional order FPK and Zakai equations use fractional integrals and derivatives. In this section we briefly introduce notions related to fractional calculus and stochastic processes, including time-changed processes. Let $g(t)$ be a continuous function on $[0, \infty)$. By definition, the fractional integral of order $\beta>0$ is

$$
J^{\beta} g(t)=\frac{1}{\Gamma(\beta)} \int_{0}^{t}(t-u)^{\beta-1} g(u) d u, \quad t>0,
$$

where $\Gamma(\cdot)$ is Euler's gamma function. By convention, $J^{0}=I$, the identity operator, and obviously, $J^{1} g(t):=J g(t)$ is the usual integration operator. The fractional derivative of order $\beta \in(0,1)$ in the sense of RiemannLiouville is

$$
D^{\beta} g(t)=\frac{d}{d t} J^{1-\beta} g(t)=\frac{1}{\Gamma(1-\beta)} \frac{d}{d t} \int_{0}^{t} \frac{g(u) d u}{(t-u)^{\beta}}, \quad t>0,
$$

and in the sense of Caputo-Djrbashian is

$$
D_{*}^{\beta} g(t)=\frac{1}{\Gamma(1-\beta)} \int_{0}^{t} \frac{g^{\prime}(u) d u}{(t-u)^{\beta}} .
$$

By convention, set $D^{\beta}=D_{*}^{\beta}=d / d t$ for $\beta=1$. These two fractional derivatives satisfy the following relationship [9]:

$$
D^{\beta} g(t)=D_{*}^{\beta} g(t)+g(0+) \frac{t^{-\beta}}{\Gamma(1-\beta)}, \quad t>0
$$

and their Laplace transforms are given by [9]:

$$
\begin{aligned}
& \widetilde{\left[D^{\beta} g\right]}(s)=s^{\beta} \tilde{g}(s)-\left(J^{(1-\beta)} g\right)(0+), \\
& {\left[\widetilde{\left.D_{*}^{\beta} g\right]}(s)=s^{\beta} \tilde{g}(s)-s^{\beta-1} g(0+),\right.}
\end{aligned}
$$

where $\tilde{g}(s) \equiv \mathcal{L}[g](s)=\int_{0}^{\infty} g(t) e^{-s t} d t$ denotes the Laplace transform of $g$.

By definition, a time-change process is a stochastic process with continuous nondecreasing sample paths starting at 0 . For details concerning general time-changed stochastic processes, see [14. Let the process $S_{t}, t \geq 0$, be a Lévy stable subordinator with the stability index $\beta \in(0,1)$, that is a stable process with $S_{0}=0$, continuous in probability, has independent increments and characteristic function $\mathbb{E}\left(e^{-\xi S_{t}}\right)=e^{-t \xi^{\beta}}$. Since $S_{t}$ is strictly increasing, its inverse process $E_{t}$ is continuous and nondecreasing, but not

a Lévy process. Further, let $E_{t}$ be the first hitting time process for $S_{t}$. The process $E_{t}$ is also called an inverse to $S_{t}$. The relation between $E_{t}$ and $S_{t}$ 
can be expressed as $E_{t}=\min \left\{\tau: S_{\tau} \geq t\right\}$. Let $f_{t}(\tau)$ be the density function of $E_{t}$ for each fixed $t>0$. If $f_{S_{1}}(t)$ is the density function of $S_{1}$, then

$$
\begin{aligned}
f_{t}(\tau) & =\frac{\partial}{\partial \tau} P\left(E_{t} \leq \tau\right)=\frac{\partial}{\partial \tau}\left(1-P\left(S_{\tau}<t\right)\right)=-\frac{\partial}{\partial \tau} P\left(S_{1}<\frac{t}{\tau^{1 / \beta}}\right) \\
& =-\frac{\partial}{\partial \tau}\left[J f_{S_{1}}\right]\left(\frac{t}{\tau^{1 / \beta}}\right)=-\frac{\partial}{\partial \tau} \int_{0}^{\frac{t}{\tau^{1 / \beta}}} f_{S_{1}}(u) d u=\frac{t}{\beta \tau^{1+\frac{1}{\beta}}} f_{S_{1}}\left(\frac{t}{\tau^{\frac{1}{\beta}}}\right) .
\end{aligned}
$$

Since $f_{S_{1}}(u) \in C^{\infty}(0, \infty)$, it follows from representation (2.7) that $f_{t}(\tau) \in$ $C^{\infty}\left(\mathbb{R}_{+}^{2}\right)$, where $\mathbb{R}_{+}^{2}=(0, \infty) \times(0, \infty)$. Further properties of $f_{t}(\tau)$ are presented in the following lemmas.

Lemma 2.1. Let $f_{t}(\tau)$ be the function given in (2.7). Then:

(a) $\lim _{t \rightarrow+0} f_{t}(\tau)=\delta_{0}(\tau)$ in the sense of the topology of the space of tempered distributions $\mathcal{D}^{\prime}(\mathbb{R})$;

(b) $\lim _{\tau \rightarrow+0} f_{t}(\tau)=\frac{t^{-\beta}}{\Gamma(1-\beta)}, t>0$;

(c) $\lim _{\tau \rightarrow \infty} f_{t}(\tau)=0, t>0$;

(d) $\mathcal{L}_{t \rightarrow s}\left[f_{t}(\tau)\right](s)=s^{\beta-1} e^{-\tau s^{\beta}}, s>0, \tau \geq 0$, where $\mathcal{L}_{t \rightarrow s}$ denotes the Laplace transform with respect to the variable $t$.

Lemma 2.2. Function $f_{t}(\tau)$ defined in (2.7) for each $t>0$ satisfies the equation

$$
D_{*, t}^{\beta} f_{t}(\tau)=-\frac{\partial}{\partial \tau} f_{t}(\tau)-\frac{t^{-\beta}}{\Gamma(1-\beta)} \delta_{0}(\tau),
$$

in the sense of tempered distributions.

For proofs of these lemmas we refer the reader to [11, 12, 24, 25]. It follows from these lemmas the following statement, which will be used in Section 3.

Corollary 2.1. The function $f_{t}(\tau)$ defined in (2.7) for each $t>0$ satisfies the equation

$$
f_{t}(\tau)=-\frac{\partial}{\partial \tau} J_{t}^{\beta} f_{t}(\tau)
$$

P r o o f. Applying the fractional integration operator $J^{\beta}$ to equation (2.8), we have

$$
f_{t}(\tau)-\lim _{t \rightarrow 0+} f_{t}(\tau)=-\frac{\partial}{\partial \tau} J_{t}^{\beta} f_{t}(\tau)-\frac{\delta_{0}(\tau)}{\Gamma(1-\beta)} J_{t}^{\beta} t^{-\beta},
$$


in the sense of distributions. Due to part (a) of Lemma 2.1 we have $\lim _{t \rightarrow 0+} f_{t}(\tau)=\delta_{0}(\tau)$. This fact together with the equation $J^{\beta} t^{-\beta}=\Gamma(1-$ $\beta$ ) implies (2.9).

Now consider a stochastic differential equation (SDE)

$$
d X_{t}=b\left(X_{t}\right) d t+\sigma\left(X_{t}\right) d B_{t}, X_{t=0}=X_{0},
$$

where $X_{0} \in \mathbb{R}^{n}$ is a random variable independent of $m$-dimensional Brownian motion $B_{t}$, the vector-function $b(x)=\left(b_{1}(x), \ldots, b_{n}(x)\right)$ and the $n \times m$ matrix-function $\sigma(x)$ satisfy the Lipschitz and growth conditions:

$$
\begin{aligned}
& \|b(x)-b(y)\|^{2}+\|\mid \sigma(x)-\sigma(y)\|^{2} \leq C_{1}\|x-y\|^{2}, \quad \forall x, y \in \mathbb{R}^{n} ; \\
& \|b(x)\|^{2}+\left.\|\sigma(x)\|\right|^{2} \leq C_{2}\left(1+\|x\|^{2}\right), \quad \forall x \in \mathbb{R}^{n},
\end{aligned}
$$

where $\|\cdot\|$ and $\||\cdot \||$ are vector- and matrix-norms, respectively. The function

$$
p(t, x)=\mathbb{E}\left[f\left(X_{t}\right) \mid X_{0}=x\right]
$$

defined as a conditional expectation of $f\left(X_{t}\right)$ under the condition $X_{0}=x$ satisfies the forward Fokker-Planck-Kolmogorov equation

$$
\begin{aligned}
\frac{\partial p(t, x)}{\partial t} & =-\sum_{j=1}^{n} \frac{\partial}{\partial x_{j}}\left(b_{j}(x) p(t, x)\right)+\frac{1}{2} \sum_{i, j=1}^{n} \frac{\partial^{2}}{\partial x_{i} \partial x_{j}}\left(a_{i, j}(x) p(t, x)\right) \\
& =-\operatorname{div}(b p)+\frac{1}{2} \operatorname{Tr}\left[Q \frac{\partial^{2} p}{\partial x^{2}}\right],
\end{aligned}
$$

on the half-space $\mathbb{R}_{+}^{n+1} \equiv\left\{(t, x) \in \mathbb{R}^{n+1}: t>0, x \in \mathbb{R}^{n}\right\}$, and the initial condition

$$
p(0, x)=f(x), x \in \mathbb{R}^{n} .
$$

In equation (2.14) $a_{i, j}(x), i, j=\ldots, n$, are entries of the matrix-function $Q(x)$, which is the product of the matrix-function $\sigma(x)$ with its transpose $\sigma^{T}(x)$. The operator on the right of $(2.14)$ is the adjoint $A^{*}$ to the second order elliptic differential operator $A$ defined as

$$
A \varphi(x)=\frac{1}{2} \sum_{i, k=1}^{n} a_{i k}(x) \frac{\partial^{2} \varphi(x)}{\partial x_{i} \partial x_{k}}+\sum_{k=1}^{n} b_{k}(x) \frac{\partial \varphi(x)}{\partial x_{k}},
$$

with the domain $\mathfrak{D}(A)=W^{1}\left(\mathbb{R}^{n}\right)$.

SDEs associated with fractional FPK equations are driven by timechanged processes. Consider SDE with a time-changed driving process

$$
d Y_{t}=b\left(X_{t}\right) d S_{t}+\sigma\left(Y_{t}\right) d B_{S_{t}}, Y_{t=0}=X_{0},
$$

We show that now the function

$$
v(t, y)=\mathbb{E}\left[f\left(Y_{t}\right) \mid Y_{0}=y\right]=\int_{0}^{\infty} f_{t}(\tau) p(\tau, y) d \tau
$$


satisfies fractional FPK equation

$$
\begin{aligned}
D_{*}^{\beta} v(t, y) & =A^{*} v(t, y) \\
& =-\operatorname{div}(b(y) v(t, y))+\frac{1}{2} \operatorname{Tr}\left[Q(x) \frac{\partial^{2} v(t, x)}{\partial x^{2}}\right],
\end{aligned}
$$

and the initial condition $v(0, y)=f(y)$. Indeed, using Lemma 2.2, one has

$$
\begin{aligned}
D_{*, t}^{\beta} v(t, x)= & \int_{0}^{\infty} D_{* t}^{\beta} f_{t}(\tau) p(\tau, x) d \tau \\
= & -\int_{0}^{\infty}\left[\frac{\partial}{\partial \tau} f_{t}(\tau)+\frac{t^{-\beta}}{\Gamma(1-\beta)} \delta_{0}(\tau)\right] p(\tau, x) d \tau \\
= & -\lim _{\tau \rightarrow \infty}\left[f_{t}(\tau) p(\tau, x)\right]+\lim _{\tau \rightarrow 0}\left[f_{t}(\tau) p(\tau, x)\right] \\
& +\int_{0}^{\infty} f_{t}(\tau) \frac{\partial}{\partial \tau} p(\tau, x) d \tau-\frac{t^{-\beta}}{\Gamma(1-\beta)} p(0, x)
\end{aligned}
$$

Due to part $(c)$ of Lemma 2.1 the first term vanishes, since $p(\tau, x)$ is bounded, while due to part $(b)$ the second and last terms cancel. Taking into account equation (2.14),

$$
D_{*, t}^{\beta} v(t, x)=\int_{0}^{\infty} f_{t}(\tau) A^{*} p(\tau, x) d \tau=A^{*} v(t, x) .
$$

Moreover, by property $(a)$ of Lemma 2.1 ,

$$
\lim _{t \rightarrow+0} v(t, x)=<\delta_{0}(\tau), p(\tau, x)>=p(0, x)=f(x) .
$$

\section{Time-changed filtering problem and fractional Zakai equation}

In the previous section we were interested in a solution conditioned on the value at the initial time $t=0$; see equation (2.13). In the filtering problem one has information of the past for all times $s, 0 \leq s<t$, coming from observations. Suppose

$$
Z_{t}=\int_{0}^{t} h\left(X_{s}\right) d s+W_{t}
$$

are $R^{m}$-valued measurements, or observations related to the process $X_{t}$ in the noisy environment. Let $\mathcal{Z}_{t}$ be a $\sigma$-algebra generated by the measurement process $Z_{t}$. One of the formulations of the filtering problem is to find the best estimation of $X_{t}$ at time $t$ in the mean square sense, given $\mathcal{Z}_{t}$. Namely, to find a stochastic process $X_{t}^{*}$ such that

$$
E\left[\left\|X_{t}-X_{t}^{*}\right\|^{2}\right]=\inf _{\left\{Y_{t}\right\}} E\left[\left\|X_{t}-Y_{t}\right\|^{2}\right],
$$

where inf is taken over all stochastic processes $Y_{t} \in L^{2}(\mathbb{P})$ under the condition that the sigma-algebra $\mathcal{Z}_{t}$ is given. It follows from the abstract theory 
of functional analysis that $X_{t}^{*}$ is the projection of $X_{t}$ onto the space of stochastic processes $\mathcal{L}\left(Z_{t}\right)=\left\{Y \in L^{2}(\mathbb{P})\right.$ : given $\left.\mathcal{Z}_{t}\right\}$. The latter can be written in the form $X_{t}^{*}=\mathbb{E}\left[f\left(X_{t}\right) \mid \mathcal{Z}_{t}\right]$, generalizing (2.13) from the initial condition $X_{0}=x$ to the entire history $\mathcal{Z}_{t}$. Hence, the filtering problem comprises of an SDE

$$
d Y_{t}=b\left(Y_{t}\right) d t+\sigma\left(Y_{t}\right) d B_{t}, Y_{t=0}=X_{0}
$$

called a state process, and an observation process

$$
d Z_{t}=h\left(Y_{t}\right) d t+d W_{t}, Z_{0}=0,
$$

obtained from (3.1) by differentiating. Brownian motion $W_{t}$ is assumed to be independent of $B_{t}$ and the initial random variable $X_{0}$. This problem was first posed in 1960th and is still under active development due to its significant applications. The linear case was studied by Kalman and Bucy [15] in the 1960th. They reduced the linear filtering problem to a linear SDE and a deterministic Riccati type differential equation. In the case of non linear filtering Kushner [17, Lipster and Shiryaev [18, and Fujisaki, Kallianpur and Kunita 7] obtained a non linear infinite dimensional stochastic differential equations for the posterior conditional density of $X_{t}$ given $\mathcal{Z}_{t}$. However, two issues arise:

(1) it is not easy to solve these equations, and

(2) it is computationally 'expensive' due to the two stage calculation procedure (prediction and correction) in the real time.

In 1969 Zakai [26] suggested a simpler way, reducing the solution of the filtering problem to a partial stochastic differential equation for the posterior unnormalized conditional density $\Phi(t, x)=p\left(t, x \mid \mathcal{Z}_{t}\right)$ for $X_{t}$. Below we briefly sketch the idea of this method. Introduce the process

$$
\rho(t)=\exp \left\{-\sum_{k=1}^{m} \int_{0}^{t} h_{k}\left(Y_{s}\right) d W_{s}-\frac{1}{2} \int_{0}^{t}\left|h\left(Y_{s}\right)\right|^{2} d s\right\}
$$

and the probability measure $d \mathbb{P}_{0}=\rho(t) d \mathbb{P}$. Further, let

$$
\Lambda_{t}=\hat{\mathbb{E}}\left(\frac{d \mathbb{P}}{d \mathbb{P}_{0}} \mid \mathcal{Z}_{t}\right)
$$

where the expectation $\hat{E}$ is under the reference measure $\mathbb{P}_{0}$. Then, as is known, the optimal filtering solution of the filtering problem (3.2), (3.3) is given by the following Kallianpur-Striebel's formula (see, e.g. [22])

$$
\mathbb{E}\left[f\left(Y_{t}\right) \mid \mathcal{Z}_{t}\right]=\frac{\hat{\mathbb{E}}\left[f\left(Y_{t}\right) \Lambda_{t} \mid \mathcal{Z}_{t}\right]}{\hat{\mathbb{E}}\left[\Lambda_{t} \mid \mathcal{Z}_{t}\right]}
$$

Moreover, under some mild conditions the unnormalized filtering measure $p_{t}(f)=\hat{\mathbb{E}}\left[f\left(Y_{t}\right) \Lambda_{t} \mid \mathcal{Z}_{t}\right]$ satisfies the following stochastic differential equation, called the Zakai equation: 


$$
p_{t}(f)=p_{0}(f)+\int_{0}^{t} p_{s}(A f) d s+\sum_{k=1}^{m} \int_{0}^{t} p_{s}\left(h_{k} f\right) d Z_{s}^{k},
$$

where $A$ is a second order elliptic differential operator given by equation (2.16). Further, introducing the filtering density $U(t, x)$ through

$$
p_{t}(f)=\int_{\mathbb{R}^{n}} f(x) U(t, x) d x,
$$

one can show that $U(t, x)$ solves the following partial stochastic differential equation (called an adjoint Zakai equation)

$$
d U(t, x)=A^{*} U(t, x) d t+\sum_{k=1}^{m} h_{k}(x) U(t, x) d Z_{k}(t),
$$

with the initial condition $U(0, x)=p_{0}(x)$. Here $A^{*}$ is the adjoint operator of $A$ defined in (2.16). Thus, if one has a solution of equation (3.8), then one will be able to establish a solution to the original filtering problem using Kallianpur-Striebel's formula (3.5). Equation (3.8) reduces to KFP equation (2.14) if the observation process $Z_{t}$ stays constant in time, which means no additional information coming from measurement/observation is used.

Now consider a filtering problem the state process and observation process of which are driven by time-changed Brownian motions. Namely, suppose the state process is

$$
d X_{t}=f\left(X_{t}\right) d T_{t}+\sigma\left(X_{t}\right) d B_{T_{t}}, X_{t=0}=X_{0},
$$

and the observation process is

$$
d Z_{t}=h\left(t, X_{t}\right) d T_{t}+d W_{T_{t}}, Z_{0}=0,
$$

where $T_{t}$ is the inverse of the Lévy stable subordinator with the stability index $\beta \in(0,1)$, and independent of $B_{t}$ and $W_{t}$. We show that the Zakai equation corresponding to this problem has the form

$$
\Phi(t, x)=p_{0}(x)+\int_{0}^{t} A^{*} \Phi(s, x) d T_{s}+\int_{0}^{t} h_{s}(x) \Phi(s, x) d Z_{T_{s}} .
$$

A few remarks before deriving this equation. The stochastic integral in equation (3.11) is well defined in the sense of Ito's integral. If $\beta \rightarrow 1$ then we recover the classical Zakai equation, since $T_{t}=t$ in this case. Hence, equation (3.11) generalizes the classic Zakai equation (3.8) for the case of filtering problem with time-changed driving processes. Note also that the time-changed process $B_{T}$ is not Markovian and has no independent increments. Therefore, the model (3.9), (3.10) can be applied to a class of correlated state processes. An important question is the existence and 
uniqueness (in an appropriate sense) of a solution for this new Zakai equation. We will discuss this question in this section as well as some solution methods useful from the application point of view.

The fractional, or time changed version of the Zakai equation in the general case of time-changed Lévy processes is obtained in paper [23] for filtering problems driven by Lévy processes. For completeness, we demonstrate the derivation of the fractional Zakai equation in our particular case of filtering problem (3.9) - (3.10). We assume that the following conditions on the input data of the filtering problem:

(C1) the vector-functions $f(x), h(x)$, and $n \times m$-matrix-function $\sigma(x)$ satisfy the Lipschitz and linear growth conditions:

$$
\begin{aligned}
\|f(x)-f(y)\|^{2} & +\|h(x)-h(y)\|^{2}+\left\||\sigma(x)-\sigma(y) \||^{2}\right. \\
& \leq C_{1}\|x-y\|^{2}, \forall x, y \in \mathbb{R}^{n} ; \\
\|b(x)\|^{2}+\|h(x)\|^{2} & +\left.\|\sigma(x)\|\right|^{2} \leq C_{2}\left(1+\|x\|^{2}\right), \forall x \in \mathbb{R}^{n},
\end{aligned}
$$

where $\|\cdot\|$ and $\||\cdot \||$ are vector- and matrix-norms, respectively.

(C2) the time-change process $T_{t}$ and Brownian motions $B_{t}$ and $W_{t}$ are independent processes;

(C3) the initial random vector $X_{0}$ is independent of processes $B_{t}, W_{t}$, and $T_{t}$ and has an infinite differentiable density function $p_{0}(x)$ decaying at infinity faster than any power of $|x|$.

Theorem 3.1. Let the conditions $(C 1)-(C 3)$ be verified. Then the filtering density $\Phi(t, x)$ associated with the filtering measure $\phi_{t}(f)$ $=\hat{\mathbb{E}}\left[f\left(X_{t}\right) \Lambda_{T_{t}} \mid \mathcal{V}_{t}\right]$, where $\mathcal{V}_{t}$ is the filtration generated by $V_{t}=Z_{T_{t}}$, satisfies the following Zakai equation

$$
\Phi(t, x)-\Phi(0, x)=\int_{0}^{t} A^{*} \Phi(s, x) d T_{s}+\sum_{k=1}^{m} \int_{0}^{t} h_{k}(x) \Phi(s, x) d Z_{T_{s}}^{(k)} .
$$

P r o o f. Let conditions (C1) - (C3) be verified. Then, in particular, the conditions for the existence of an unnormalized filtering distribution $p_{t}(f)=\hat{\mathbb{E}}\left[f\left(Y_{t}\right) \Lambda_{t} \mid \mathcal{Z}_{t}\right]$ which solves the Zakai equation (3.6), is also verified. Here $Y_{t}$ is a solution to stochastic differential equation (3.2). According to Theorem 3.3 in [12 the time-changed process $X_{t}=Y_{T_{t}}$ solves stochastic differential equation (3.9).

The connection $X_{t}=Y_{T_{t}}$ between the state processes $X_{t}$ and $Y_{t}$ implies the connection $V_{t}=Z_{T_{t}}$ between the observation processes $V_{t}$ and $Z_{t}$. Indeed, letting $T_{t}=\tau$, or the same $D_{\tau}=t$, one obtains from the relation $d V_{t}=h\left(Y_{T_{t}}\right) d T_{t}+d W_{T_{t}}$ and (3.3) that $Z_{\tau}=V_{D_{\tau}}$, or the same $V_{t}=Z_{T_{t}}$. It 
follows that the filtration $\mathcal{V}_{t}$ coincides with the filtration $\mathcal{Z} \circ \mathcal{T}_{t} \equiv \mathcal{Z}_{\mathcal{T}_{t}}$ generated by the time changed observation process $Z_{T_{t}}$. Hence, the unnormalized filtering distribution $\phi_{t}(f)=\hat{\mathbb{E}}\left[f\left(X_{t}\right) \Lambda_{T_{t}} \mid \mathcal{Z} \circ \mathcal{T}_{t}\right]$ corresponding to the filtering problem (3.9), (3.10) is the time-changed process $\phi_{t}(f)=p_{T_{t}}(f)$. Therefore, due to equation (3.1) the process $\phi_{t}(f)$ satisfies

$$
\phi_{t}(f)=p_{T_{t}}(f)=p_{0}(f)+\int_{0}^{T_{t}} p_{s}(A f) d s+\sum_{k=1}^{m} \int_{0}^{T_{t}} p_{s}\left(h_{k} f\right) d Z_{s}^{(k)} .
$$

Further, using the change of variable formula (see [14, Proposition 10.21) $\int_{0}^{T_{t}} H_{s} d S_{s}=\int_{0}^{t} H_{T_{s-}} d S_{T_{s}}$, for stochastic integrals driven by a semimartingale $S_{t}$, we obtain

$$
\begin{aligned}
\int_{0}^{T_{t}} p_{s}(A f) d s & =\int_{0}^{t} \hat{\mathbb{E}}\left[A f\left(Y_{T_{s}}\right) \Lambda_{T_{s}} \mid \mathcal{Z}_{T_{s}}\right] d Z_{T_{s}}^{(k)}=\int_{0}^{t} \hat{\mathbb{E}}\left[A f\left(X_{s}\right) \Lambda_{T_{s}} \mid \mathcal{Z}_{T_{s}}\right] d Z_{T_{s}}^{(k)} \\
& =\int_{0}^{t} \phi_{s}(A f) d Z_{T_{s}}^{(k)}
\end{aligned}
$$

and

$$
\begin{aligned}
\sum_{k=1}^{m} \int_{0}^{T_{t}} p_{s}\left(h_{k} f\right) d Z_{s}^{(k)} & =\sum_{k=1}^{m} \int_{0}^{t} \hat{\mathbb{E}}\left[h_{k}\left(Y_{T_{s}}\right) f\left(Y_{T_{s}}\right) \Lambda_{T_{s}} \mid \mathcal{Z}_{T_{s}}\right] d Z_{T_{s}}^{(k)} \\
& =\sum_{k=1}^{m} \int_{0}^{t} \hat{\mathbb{E}}\left[h_{k}\left(X_{s}\right) f\left(X_{s}\right) \Lambda_{T_{s}} \mid \mathcal{Z}_{T_{s}}\right] d Z_{T_{s}}^{(k)} \\
& =\sum_{k=1}^{m} \int_{0}^{t} \phi_{s}\left(h_{k} f\right) d Z_{T_{s}}^{(k)} .
\end{aligned}
$$

Equations (3.13), (3.14), and (3.15) imply the desired equation (3.12).

Let $T_{t}$ be the inverse to a stable Lévy subordinator $D_{t}$ of a stability index $\beta \in(0,1)$ and let the stochastic processes $\Pi_{t}(f)$ and $\Pi_{t, Z}(f)$ are defined by

$\Pi_{t}(f)=A p_{t}(f)=\int_{0}^{\infty} g_{t}(\tau) p_{\tau}(f) d \tau, \Pi_{t, Z}(f)=C p_{t}(f)=\int_{0}^{\infty} g_{t}(\tau) p_{\tau}(f) d Z_{\tau}$,

where $g_{t}(\tau)$ is the density function of the process $T_{t}$ and $p_{t}(f)$ is the unnormalized filtering distribution of the Zakai equation (3.6) corresponding to the filtering model (3.2)-(3.3). Then it follows from equation (3.12) that the following stochastic relation holds:

$$
\Pi_{t}(f)-p_{0}(f)=J_{t}^{\beta}\left(\Pi_{t}(A f)+\sum_{k=1}^{m} \Pi_{t, Z^{(k)}}\left(h_{k} f\right)\right),
$$

where $J_{t}^{\beta}$ is the fractional integration operator of order $\beta$. 
Let $B$ maps the class of stochastic processes $\Pi_{t}(f)$ to the class of processes $\Pi_{t, Z}(f)$, that is $\Pi_{t, Z}(f)=B \Pi_{t}(f)$. One can verify easily that the operator $B$ can be expressed with the help of operators $A$ and $C$ in equation (3.16). Namely,

$$
B=C A^{-1} \text {. }
$$

Using $L^{2}(\mathbb{P})$-norm and calculus of stochastic processes one can show that $A$ is a one-to-one bounded linear operator and $C$ is a bounded linear operator. Therefore, it follows that operator $B$ is well defined bounded linear operator. We note that equation (3.17) can be written in the form

$$
\Pi_{t}(f)-p_{0}(f)=J_{t}^{\beta}\left(\Pi_{t}(A f)+\sum_{k=1}^{m} B_{k} \Pi_{t}\left(h_{k} f\right)\right)
$$

where

$$
B_{k} \Pi_{t}(f)=\Pi_{t, Z^{(k)}}(f) .
$$

The differential form of (3.17) involves a fractional derivative in the RiemannLiuoville sense

$$
d \Pi_{t}(f)=\mathcal{D}_{t}^{1-\beta} \Pi_{t}(A f) d t+\sum_{k=1}^{m} \mathcal{D}_{t}^{1-\beta} B_{k} \Pi_{t}\left(h_{k} f\right) d t, \Pi_{t=0}(f)=p_{0}(f) .
$$

The latter in terms of unnormalized densities associated with the process $\Pi_{t}(f)$ can be represented in the form

$$
D_{*}^{\beta} U(t, x)=A^{*} U(t, x)+\sum_{k=1}^{m} h_{k}(x) B_{k} U(t, x), U(0, x)=f(x),
$$

where $D_{*}^{\beta}$ is the fractional derivative in the sense of Caputo. Equation (3.22) generalizes fractional FPK equation (2.19) to the case of fractional filtering problems.

Theorem 3.2. Let the conditions $(C 1)-(C 3)$ be verified. Then there exists a unique filtering density $\Phi(t, x)$ satisfying the fractional Zakai equation (3.12). Moreover, there exist uniquely defined stochactioc processes in equation (3.16) satisfying initial value problem (3.21).

P r o o f. Suppose there are two filtering densities $\Phi_{1}(t, x)$ and $\Phi_{2}(t, x)$ such that both satisfy equation (3.12). Then the process $\Psi(t, x)=\Phi_{2}(t, x)-$ $\Phi_{1}(t, x)$ satisfies the following equation

$$
\Psi(t, x)=\int_{0}^{t} A^{*} \Psi(s, x) d T_{s}+\sum_{k=1}^{m} \int_{0}^{t} h_{k}(x) \Psi(s, x) d Z_{T_{s}}^{(k)} .
$$


This ia a SDE driven by time-changed processes $T_{s}$ given $Z_{T_{s}}$. Its counterpart with non time-changed process has the form

$$
V(t, x)-V_{0}(x)=\int_{0}^{t} A^{*} V(s, x) d s+\sum_{k=1}^{m} \int_{0}^{t} h_{k}(x) V(s, x) d Z_{s}^{(k)},
$$

with $V_{0}(x) \equiv 0$. The solutions $V(t, x)$ and $\Psi(t, x)$ are related through $\Psi(t, x)=V\left(T_{t}, x\right)$. Equation (3.24) has a unique solution (see, e.g. [10, 8, 21, [16]). Since the initial condition is $V(0, x)=0$, then the corresponding solution $V(t, x) \equiv 0$ in the sense of $L^{2}(\mathbb{P})$. This implies $\Psi(t, x) \equiv 0$, or the same, $\Phi_{1}(t, x)=\Phi_{2}(t, x)$ in the sense of $L^{2}(\mathbb{P})$. The latter, in turn, implies that the process defined in equation (3.16) is unique.

\section{On some solution methods}

Knowing a solution of a nonlinear filtering problem one can use it for solution of the associated fractional nonlinear filtering problem. Two approaches to the solution of nonlinear filtering problems are commonly used. Namely,

(1) direct solution of filtering problem (3.9)-(3.10).

(2) solution of the Zakai equation followed by the Kallianpur-Striebel formula.

For the filtering problem with no time-changed driving processes both approaches are well studied; see, e.g. works [16, 22, 3, 13] for the first approach, and [26, 1, 2, 20, 19] for the second approach. Below we briefly sketch the ideas of some methods of solution of nonlinear filtering problems (first approach) and of fractional Zakai type equations (3.12) and (3.21) (second approach). Both type of Zakai equations (3.12) and (3.21) (or its equivalent form (3.22) ) are of great interest in various applications of fractional filtering problems. For solution of these equations the following formulas are important:

$$
\Phi_{t}(f)=p_{T_{t}}(f)
$$

and

$$
\Pi_{t}(f)=\int_{0}^{\infty} g_{t}(\tau) p_{\tau}(f) d \tau .
$$

Therefore, in the first step one needs to find the stochastic process $p_{t}(f)$ which solves the classical Zakai equation. Then using the above formulas one can find solutions to fractional Zakai equations. Like the non-fractional case, one can develop analytic and numerical methods, and methods for solution of filtering problem directly, or through the associated Zakai equation. Accordingly, in the fractional case the methods can be developed for solution of three following situations: 
(i) Direct solution of the fractional filtering problem;

(ii) Solution of the fractional filtering problem through the Zakai equation (3.12);

(iii) Solution of the fractional filtering problem through the Zakai equation (3.22).

Case (i). If $Y_{t}$ is a solution of the filtering problem with the state process (3.2) and the observation process (3.3), then the process $X_{t}=Y_{T_{t}}$ solves the fractional filtering problem (3.9)-(3.10) (see the proof of Theorem 3.1). Therefore, if $p(t, x)$ is the conditional density of $Y_{t}$ then the conditional density of $X_{t}$ is calculated by conditioning on $T_{t}$, that is

$$
\begin{aligned}
\tilde{p}(t, x) & =f_{X_{t} \mid \mathcal{Z}_{T_{t}}}(x)=\int_{0}^{\infty} p(\tau, x) \mathbb{P}\left(T_{t} \in d \tau\right) \\
& =\int_{0}^{\infty} g_{t}(\tau) p(x, \tau) d \tau .
\end{aligned}
$$

Case (ii). In this case the unique solution $\Phi(t, x)$ to the fractional Zakai equation (3.12) is $\Phi(t, x)=V\left(T_{t}, x\right)$, where $V(t, x)$ is an unnormalized solution to the Zakai equation

$$
d V(t, x)=A^{*} V(t, x) d t+\sum_{k=1}^{m} h_{k}(x) V(t, x) d Z_{t}^{(k)}, V(0, x)=f(x) .
$$

Again, like Case (i) the expectation of $\Phi(t, x)$ can be computed using the formula

$$
\begin{aligned}
\mathbb{E}(\Phi(t, x)) & =\mathbb{E}\left(V\left(T_{t}, x\right)\right)=\int_{0}^{\infty} \mathbb{E}(V(\tau, x)) \mathbb{P}\left(T_{t} \in d \tau\right) \\
& =\int_{0}^{\infty} g_{t}(\tau) \mathbb{E}(V(\tau, x)) d \tau .
\end{aligned}
$$

Case (iii). In this case, it follows from the first equation in (3.16), that the unique solution to the fractional Zakai equation (3.22) can be represented as

$$
U(t, x)=\int_{0}^{\infty} V(\tau, x) \mathbb{P}\left(T_{t} \in d \tau\right)=\int_{0}^{\infty} g_{t}(\tau) V(\tau, x) d \tau .
$$

Thus, in all three cases (i)-(iii) for solutions of fractional filtering problem we obtain the same type integral (see equations (4.3), (4.4), and (4.5)) $\int_{0}^{\infty} g_{t}(\tau) G(\tau, x) d \tau$, where $G(t, x)$ is a stochastic process, which solves an Itto type SDE (non fractional filtering problem). The details of calculation of integrals (4.3), (4.4), and (4.5) for solution of fractional filtering problem and fractional Zakai equation will be discussed in a separate paper. 


\section{On the particle flow method for fractional filtering problems}

Due to the Bayes rule for the posterior conditional density one has

$$
p(x, \lambda)=\frac{g(x) h(x)^{\lambda}}{K(\lambda)}, K(0)=1,
$$

where $g(x)$ is the prior, $h(x, \lambda)$ is the likelihood, and $K(\lambda)$ is the normalizing factor. Obviously, $\lambda=0$ corresponds to the prior $p(x, 0)=g(x)$ and $\lambda=1$ gives the posterior $p(x, 1)=\frac{g(x) h(x)}{K(1)}$. In $\log$ terms this equation takes the form

$$
\ln p(x, \lambda)=\ln g(x)+\lambda \ln h(x)-\ln K(\lambda) .
$$

If the flow obeys SDE $d \mathbf{X}=\mathbf{f}(\mathbf{X}, \lambda) d \lambda+d W$, where $W$ is a Brownian motion with the covariance matrix $Q$, then the density $p(x, \lambda)$ of $\mathbf{X}$ satisfies the Fokker-Planck equation

$$
\frac{\partial p}{\partial \lambda}=-\operatorname{div}(\mathbf{f} p)+\frac{1}{2} \operatorname{Tr}\left[Q \frac{\partial^{2} p}{\partial x^{2}}\right]
$$

Combining equations (5.1) and (5.2) one has a system of PDEs

$$
\operatorname{div} \mathbf{q}=\eta_{0}
$$

where

$$
\mathbf{q}=\mathbf{f} p \text { and } \eta_{0}(\lambda, x)=\operatorname{Tr}\left[Q \frac{\partial^{2} p(\lambda, x)}{\partial x^{2}}\right]-p(\lambda, x)\left[\ln h(x)-\frac{d \ln K(\lambda)}{d \lambda}\right] .
$$

At this stage various possibilities arise as discussed in series of papers by $\mathrm{F}$. Daum and co-workers (see, e.g. [4, 5] and references therein). For instance, if one assumes that there is no diffusion $(Q=0)$, then $\eta$ becomes

$$
\eta_{0}(\lambda, x)=-p(\lambda, x)\left[\ln h(x)-\frac{d \ln K(\lambda)}{d \lambda}\right] .
$$

It is well-known from the general theory of of PDEs that equation (5.3) has a solution only if the right hand side $\eta_{0}$ is orthogonal to 1 , that is

$$
\int_{\Omega} \eta_{0}(\lambda, x) d x=0 .
$$

Here $\Omega$ is the range of the random vector $X_{t}$.

Let $\phi(x, \lambda)$ is a conditional density of the fractional filtering problem: $\phi(x, \lambda)=f_{X_{\lambda} \mid \mathcal{Z}_{T_{\lambda}}}(x) ; g(x)$ is the prior density, $h(x)$ is the likelihood, and $K(\lambda)$ is the normalization factor. Then the Bayes rule implies

$$
\ln \phi(x, \lambda)=\ln g(x)+\lambda \ln h(x)-\ln K(\lambda) .
$$

In this case the flow obeys $\operatorname{SDE} d \mathbf{X}_{\lambda}=\mathbf{f}\left(\mathbf{X}_{\lambda}, \lambda\right) d T_{\lambda}+d W_{T_{\lambda}}$, where $T$ is a time-change process, $W$ is a Brownian motion with the covariance matrix 
$Q$. Then the density $\phi(x, \lambda)$ of $\mathbf{X}$ satisfies the stochastic differential equation (3.22), that is

$$
D_{*}^{\beta} \phi(\lambda, x)=A^{*} \phi(\lambda, x)+\sum_{k=1}^{m} h_{k}(x) B_{k} \phi(\lambda, x), \phi(0, x)=\delta_{0}(x),
$$

where $D_{*}^{\beta}$ is the fractional derivative in the sense of Caputo, $A^{*}$ is the adjoint operator to $A$, defined in (2.16), and operators $B_{k}$ are defined in (3.20).

We first consider the case of particle flow without diffusion. This case for non fractional filtering was discussed in papers [4, 5]. It is obvious, that if there is no noise, then $B_{k} \equiv 0$, and equation (5.8) takes the form

$$
D_{*}^{\beta} \phi(\lambda, x)=-\operatorname{div}(f(\lambda, x) \phi(\lambda, x)), \phi(0, x)=\delta_{0}(x),
$$

The left side of (5.9) due to equation (5.7) equals

$$
\begin{aligned}
D_{*}^{\beta} \phi(\lambda, x) & =J^{1-\beta} \frac{d \phi(\lambda, x)}{d \lambda}=J^{1-\beta}\left[\phi(\lambda, x)\left(\ln h(x)-\frac{d \ln K(\lambda)}{d \lambda}\right)\right] \\
& =\frac{1}{\Gamma(1-\beta)} \int_{0}^{\lambda}(\lambda-\mu)^{-\beta}\left[\phi(\mu, x)\left(\ln h(x)-\frac{d \ln K(\mu)}{d \mu}\right)\right] d \mu
\end{aligned}
$$

where $\Gamma(\cdot)$ is the Euler gamma function. Therefore, the problem of particle flow reduces to the following partial differential equation

$$
\operatorname{div}(\phi f(\lambda, x))=\eta(\lambda, x),
$$

where

$$
\eta(\lambda, x)=-\frac{1}{\Gamma(1-\beta)} \int_{0}^{\lambda}(\lambda-\mu)^{-\beta}\left[\phi(\mu, x)\left(\ln h(x)-\frac{d \ln K(\mu)}{d \mu}\right)\right] d \mu .
$$

Equation (5.12) generalizes the form (5.5) for the function $\eta_{0}(\lambda, x)$, coinciding with it if $\beta=1$. We note that if the function $\eta_{0}(\lambda, x)$ in (5.5) satisfies the orthogonality condition (5.6) then the function $\eta(\lambda, x)$ in equation (5.12) also satisfies condition (5.6). Indeed, due to the Fubini theorem, one has

$$
\int_{\Omega} \eta(\lambda, x) d x
$$




$$
\begin{aligned}
& =-\frac{1}{\Gamma(1-\beta)} \int_{\Omega}\left(\int_{0}^{\lambda}(\lambda-\mu)^{-\beta}\left[\phi(\mu, x)\left(\ln h(x)-\frac{d \ln K(\mu)}{d \mu}\right)\right] d \mu\right) d x \\
& =-\frac{1}{\Gamma(1-\beta)} \int_{0}^{\lambda}(\lambda-\mu)^{-\beta} \int_{\Omega}\left[\phi(\mu, x)\left(\ln h(x)-\frac{d \ln K(\mu)}{d \mu}\right)\right] d x d \mu \\
& =-\frac{1}{\Gamma(1-\beta)} \int_{0}^{\lambda}(\lambda-\mu)^{-\beta}\left(\int_{\Omega} \eta_{0}(\mu, x) d x\right) d \mu=0,
\end{aligned}
$$

since $\phi(\lambda, x)=p(\lambda, x)$. Therefore, equation (5.11) has a solution. Equation (5.11) is an underdetermined equation, and therefore, has no unique solution. Depending on researcher's interest it can be found an appropriate solution.

In the general case with non-zero diffusion, the right hand side of equation (5.12) has the form

$$
\begin{aligned}
\eta(\lambda, x) & =\frac{1}{\Gamma(1-\beta)} \int_{0}^{\lambda}(\lambda-\mu)^{-\beta}\left[\operatorname{Tr}\left[Q \frac{\partial^{2} p(\lambda, x)}{\partial x^{2}}\right]\right. \\
& \left.-\phi(\mu, x)\left(\ln h(x)-\frac{d \ln K(\mu)}{d \mu}\right)\right] d \mu,
\end{aligned}
$$

generalizing equation (5.4). The PDE (5.11) can be simplified similar to non-fractional case, see [4, 5].

\section{References}

[1] A. Bensoussan, R. Glowinski, A. Rascanu, Approximations of Zakai equation by the splitting up method. SIAM J. Control Optim. 28, No 6 (1989), 1420-1431.

[2] A. Budhiraja, G. Kallianpur, Approximation to the solutions of Zakai equations using multiple Wiener and Stratonovich expansions. Stochastics 56 (1996), 271-315.

[3] F. Daum, Solution of the Zakai equation by separation of variables. IEEE Trans. on Automatic Control AC-32, No 10 (1987), 941-943.

[4] F. Daum, J. Huang, Particle flow with non-zero diffusion for nonlinear filters, Bayesian decision, and transport. In: Proc. of SPIE Conf. on Signal Processing (Ed. Ivan Kadar), Baltimore, April (2013).

[5] F. Daum, J. Huang, A. Noushin, New theory and numerical experiments for Gromov's method with stochastic particle flow. In: Proc. of IEEE FUSION Conference, Cambridge, UK, July (2018). 
[6] A. Einstein, Über die von der molekularkinetischen Theorie der Wärme geforderte Bewegung von in ruhenden Flüssigkeiten suspendierten Teilchen [Engl. Transl.: On the motion of small particles suspended in a stationary liquid, as required by the molecular kinetic theory of heat]. Annalen der Physic 17, No 8 (1905), 549-560.

[7] M. Fujisaki, G. Kallianpur, H. Kunita, Stochastic differential equations for the nonlinear filtering problem. Osaka J. of Mathematics 9, No 1 (1972), 19-40.

[8] B. Grigelionis, Stochastic non-linear filtering equations and semimartingales. In: Lecture Notes Math. 972 (Eds. S.K. Mitter, A. Moro), Springer-Verlag, Berlin (1982), 63-99.

[9] R. Gorenflo, F. Mainardi, Fractional calculus: Integral and differential equations of fractional order. In: A. Carpinteri and F. Mainardi (Eds.): Fractals and Fractional Calculus in Continuum Mechanics, Springer (1997), 223-276.

[10] I. Gyöngy, N.V. Krylov, SPDE's with unbounded coefficients and applications, I, II. Stochastics and Stochastic Reports 32 (1990), 53-91, $165-180$.

[11] M. Hahn, S. Umarov, Fractional Fokker-Plank-Kolmogorov type equations and their associated stochastic differential equations. Fract. Calc. Appl. Anal. 14, No 1 (2011), 56-79; DOI: 10.2478/s13540-011-0005-9; https://www.degruyter.com/view/j/fca.2011.14.issue-1/ issue-files/fca.2011.14.issue-1.xml.

[12] M. Hahn, K. Kobayashi, S. Umarov, Fokker-Planck-Kolmogorov equations associated with time-changed fractional Brownian motion. Proc. Amer. Math, Soc. 139, No 2 (2010), 691-705; DOI: 00029939(2010)10527-0.

[13] K. Itô, K. Xiong, Gaussian filters for nonlinear filtering problems. IEEE Trans. on Automatic Control 45, No 5 (2000), 910-927.

[14] J. Jacod, Calcul Stochastique et Problèmes de Martingales. In: Lecture Notes in Mathematics 714, Springer, Berlin (1979), 539 pp.

[15] R.E. Kalman, R.S. Bucy, New results in linear filtering and prediction theory. J. of Basic Engineering 83 (1961), 95-108.

[16] H. Kunita, Stochastic Flows and Stochastic Differential Equations. Cambridge University Press, Cambridge (1990).

[17] H.J. Kushner, Dynamical equations for optimal nonlinear filtering. J. of Differential Equations 3 (1967), 179-190.

[18] R.Sh. Lipster, A.N. Shiryaev, Statistics of Random Processes, I, II. Springer, New York (2002). 
[19] S. Lototsky, R. Mikulevicius, R. Rozovskii, Nonlinear filtering revisited: A spectral approach. SIAM J. Control Optimization 35 (1997), 435-461.

[20] T. Meyer-Brandis, F. Proske, Explicit solution of a non-linear filtering problem for Lévy processes with applications to finance. Appl. Math. Optim. 50 (2004), 119-134.

[21] E. Pardoux, Stochastic differential equations and filtering of diffusion processes. Stochastics 3 (1979), 127-167.

[22] B.L. Rozovskii, Stochastic Evolution Systems. Linear Theory and Applications to Nonlinear Filtering. Kluwer Academic Publishers, Dotrecht (1990).

[23] S. Umarov, F. Daum, K. Nelson, Fractional generalizations of filtering problems and their associated fractional Zakai equations. Fract. Calc. Appl. Anal. 17, No 3 (2014), 745-764; DOI: 10.2478/s13540-014-0197x; https://www.degruyter.com/view/j/fca.2014.17.issue-3/

issue-files/fca.2014.17.issue-3.xml.

[24] S. Umarov, Introduction to Fractional and Pseudo-Differential Equations with Singular Symbols. Ser. Developments in Mathematics, 41, Springer (2015).

[25] S. Umarov, M. Hahn, K. Kobayashi, Beyond the Triangle: Brownian Motion, Itô Calculus, and Fokker-Planck Equation - Fractional Generalizations. World Scientific (2018).

[26] M. Zakai, On the optimal filtering of diffusion processes. Z. Wahrsch. Verw. Gebiete 11, No 3 (1969), 230-243.

1 University of New Haven, Department of Mathematics

300 Boston Post Road

West Haven, CT 06516, USA

e-mail: sumarov@newhaven.edu

Received: December 22, 2017

2 Raytheon, 235 Presidents Way

Woburn, MA, USA

e-mail:daum@raytheon.com

3 Boston University, Electrical and Computer Engineering

8 Saint Mary's St.,

Boston, MA 02215, USA

e-mail: kenricpn@bu.edu

Please cite to this paper as published in:

Fract. Calc. Appl. Anal., Vol. 21, No 2 (2018), pp. 336-353,

DOI: 10.1515/fca-2018-0020 